\title{
SOME PROBLEMATIC CASES OF VP ELLIPSIS
}

\author{
Daniel Hardt \\ Department of Computer and Information Science \\ University of Pennsylvania \\ Philadelphia, PA 19104 \\ Internet: hardt@linc.cis.upenn.edu
}

\section{INTRODUCTION}

It has been widely assumed that VP ellipsis is governed by an identity condition: the elided VP is interpreted as an identical copy of another expression in surrounding discourse. For example, Sag (76) imposes an identity condition on Logical Form representations of VP's. A basic feature of this account is the requirement that a syntactic VP be available as the antecedent. This requirement is reflected in most subsequent accounts as well. In this paper I examine three cases of VP ellipsis in which the antecedent cannot be identified with any VP. These cases, which are illustrated using naturallyoccurring examples, present a fundamental problem for any of the standard approaches. I will argue that they receive a natural treatment in the system I have developed, in which VP ellipsis is treated by storing VP meanings in a discourse model.

I will address the following three problems:

- Combined Antecedents: The antecedent may be a combination of more than one previous property.

- Passive Antecedents: the antecedent in a passive clause may not be associated with any VP, but, rather, the property associated with the active counterpart of that clause.

- NP Antecedents: the antecedent may be a property associated with an NP.

In what follows, I sketch my general approach to VP ellipsis, after which I show how each of the above phenomena can be treated in this approach.

\section{BACKGROUND}

VP ellipsis, I suggest, is to be explained along the lines of familiar accounts of pronominal anaphora (e.g., Kamp 80, Heim 81). A discourse model is posited, containing various semantic objects, including (among other things) entities and properties that have been evoked in preceding discourse. Typically, entities are evoked by NP's, and properties by VP's. The interpretation of a pronoun involves a selection among the entities stored in the discourse model. Similarly, the interpretation of an elliptical VP involves a selection among the properties stored in the discourse model. ${ }^{1}$ I have described an implementation along these lines in Hardt 91, based on some extensions to the Incremental Interpretation System (Pereira and Pollack 91).

There are two rules governing VP ellipsis: one allowing the introduction of properties into the discourse model, and another allowing the recovery of properties from the discourse model.

These two rules are given below. In general, I assume the form of grammar in Pereira and Pollack 91 , in which all semantic rules take the input and output discourse models as arguments. That is, all semantic rules define relations on discourse models, or "file change potentials", in Heim's terms.

The (simplified) rule for recovering a property from the discourse model is:

$$
\begin{aligned}
& \mathrm{AUX} \Rightarrow \mathrm{P} \\
& \text { where } \mathrm{P} \epsilon \mathrm{DM}_{\text {in }} .
\end{aligned}
$$

That is, an auxiliary verb is replaced by some property $\mathbf{P}$ stored in the input discourse model.

Secondly, properties are introduced into the discourse model by the following rule:

Upon encountering a property-denoting semantic object of the form:

$$
\mathrm{P}\left(-, \mathbf{a}_{1}\right)
$$

that is, a predicate with the first argument slot empty, we have:

$$
\mathrm{DM}_{\text {out }}=\mathrm{DM}_{\text {in }} \cup\left\{\mathrm{P}\left(-, \mathrm{a}_{1}\right)\right\}
$$

This indicates that the property is added to the output discourse model. Typically, the propertydenoting expression is associated with a VP, although other types of expressions can also introduce properties into the discourse model.

I have argued elsewhere (Hardt 91, 91a) that such a system has certain important advantages over alternative approaches, such as those of Sag (76) and Williams (77). ${ }^{2}$

\footnotetext{
${ }^{1}$ To be precise, it is not properties that are stored as VPE antecedents, but relations involving an input and output discourse context as well as a property.

${ }^{2}$ The DRT-based account of Klein (87) essentially du-
} 
In what follows, I will briefly examine the phenomena listed above, which present fundamental problems for all accounts of VP ellipsis of which I am aware ${ }^{3}$. For each problem, I will suggest that the current approach provides a solution.

\section{COMBINED ANTECEDENTS}

There are cases of VP ellipsis in which the antecedent is combined from two or more separate VP's. This presents a problem for most accounts of VP ellipsis, since there is no syntactic object consisting of the combination of two separate VP's. If antecedents are stored in the discourse model, as I am suggesting, this is not surprising. For example, it is well known that combinations of entities can become the antecedent for a plural pronoun. Consider the following example:

After the symmetry between left-handed particles and right-handed anti- particles was broken by the kaons in the 1960 s, a new symmetry was introduced which everybody swears is unbreakable. This is between left-handed particles moving forwards in time, and righthanded anti-particles moving backwards in time (none do, in any practical sense, but that does not worry theorists too much).

From: The Economist, \& August 1990, p.69. Bonnie Webber, p.c.

The meaning of the elided VP ("none do") is, I take it, "none do move forwards or move backwards in time". So the antecedent must consists of a combination of properties associated with two VP's: "moving forwards in time" and "moving backwards in time".

Such an example indicates the necessity for a rule allowing the set of properties in the discourse model to be expanded, as follows:

$$
\{P \ldots Q \ldots\} \Rightarrow\{P \ldots Q \ldots[P \text { OP Q }]\}
$$

That is, if the discourse model contains two properties $\mathbf{P}$ and $\mathrm{Q}$, it may also contain the property resulting from a combination of $P$ and $Q$ by some operator (I assume that the operators include AND and $\mathrm{OR}$ ).

Another example is the following:

So I say to the conspiracy fans: leave him alone. Leave us alone. But they won't.

From: The Welcomat, $5 \mathrm{Feb}$ 92, p.25

Here the meaning of the elliptical VP is: "they won't leave him alone or leave us alone".

plicates the Sag/Williams approach in DRT. Of particular relevance here is Klein's requirement that the antecedent be a DRT-representation of a syntactic VP.

${ }^{3}$ The recent account of Dalrymple, Shieber and Pereira (91) does treat the "Passive Antecedent" problem. However, no treatment of the "Combined Antecedent" or "NP Antecedent" problems is given.
This phenomenon has been noted in the literature, in particular by Webber (78), in which the following examples were given:

I can walk, and I can chew gum. Gerry can too, but not at the same time.

Wendy is eager to sail around the world and Bruce is eager to climb Kilimanjaro, but neither of them can because money is too tight.

By the rule given above, this example could be given the interpretation "neither of them can sail around the world or climb Kilimanjaro".

It is clear that the combining operation is highly constrained. In all the examples mentioned, either $P$ and $Q$ have the same subject, or the subject of the elliptical VP refers to the two subjects of $P$ and Q. In future work, I will attempt to formulate constraints on this operation.

\section{PASSIVE ANTECEDENTS}

The next problem is illustrated by the following example, cited by Dalrymple, Shieber and Pereira (91):

A lot of this material can be presented in a fairly informal and accessible fashion, and often I do.

From: Noam Chomsky on the Generative Enterprise, Foris Publications, Dordrecht. 1982.

The antecedent for the elliptical VP is "present a lot of this material in a fairly informal and accessible fashion". This is not associated with a VP, although the active counterpart of the sentence would contain such a VP. This is not surprising from a semantic point of view, since it is traditionally held that a 'passive transformation' preserves semantic equivalence.

\section{Another example of this is following:}

Business has to be developed and defended differently than we have in the past. From: NPR interview, 24 May 91

The most straightforward treatment of such phenomena in the current framework is to assume that the syntactic derivation of a passive antecedent such as "this material can be presented" corresponds to a semantic object

present(-, this material)

More generally, for a syntactic expression

SUBJ be VPten

the corresponding semantic object is

VP' $(-$, SUBJ')

That is, the denotation of the "surface subject" becomes the second argument of the VP-denotation. This semantic object, then, satisfies the condition on the rule for introducing properties given above. 
Thus, under such a treatment of the passive, these examples are accommodated in the present system without further stipulations.

\section{NP ANTECEDENTS}

In many cases, the antecedent property is introduced by a NP rather than a VP. This would be difficult to explain for a syntactic or logical form theory. From a semantic point of view, it is not surprising, since many NP's contain a common noun which is standardly analyzed semantically as denoting a property. Consider the following (naturally occurring) example:

We should suggest to her that she officially appoint us as a committee and invite faculty participation/input. They won't, of course,...

From: email message. (Bonnie Webber, p.c.)

In this example, the meaning of the elided VP is "they won't participate". The source is the NP "faculty participation".

Another example is the following:

[Many Chicago-area cabdrivers] say their business is foundering because the riders they depend on - business people, downtown workers and the elderly - are opting for the bus and the elevated train, or are on the unemployment line. Meanwhile, they sense a drop in visitors to the city. Those who do, they say, are not taking cabs.

From: Chicago Tribune front page, 2/6/92. Gregory Ward, p.c.

Here, the meaning of the elided VP is "those who do visit", where the source is the NP "visitors".

In the current framework, such examples could be treated as follows. Assume, following Chierchia (84), that there is a class of nouns that are semantically correlated with properties. For any such noun, the associated property can be added to the discourse model, just as is done for verbs.

\section{CONCLUSIONS}

The cases investigated constitute strong evidence that VP ellipsis must be explained at a semantic/discourse level. I have argued that the examples can be dealt with in the system I have developed. In future work, I will formulate constraints on the operations described here.

\section{ACKNOWLEDGEMENTS}

Thanks to Aravind Joshi, Shalom Lappin, Gregory Ward, and Bonnie Webber. This work was supported by the following grants: ARO DAAL 0389-C-0031, DARPA N00014-90-J-1863, NSF IRI 9016592, and Ben Franklin 91S.3078C-1.

\section{REFERENCES}

Gennaro Chierchia. Formal Semantics and the Grammar of Predication. Linguistic Inquiry, Vol. 16, no. 3. Summer 1984.

Mary Dalrymple, Stuart Shieber and Fernando Pereira. Ellipsis and Higher-Order Unification. Linguistics and Philosophy. Vol. 14, no. 4, August 1991.

Daniel Hardt. A Discourse Model Account of VP Ellipsis. Proceedings AAAI Symposium on Discourse Structure in Natural Language Understanding and Generation. Asilomar, CA., November 1991.

Daniel Hardt. Towards a Discourse Model Account of VP Ellipsis. Proceedings ESCOL 1991. Baltimore, MD.

Irene Heim. The Semantics of Definite and Indefinite Noun Phrases. Ph.D. thesis, University of Massachusetts-Amherst. 1981.

Hans Kamp. A Theory of Truth and Semantic Representation. In Groenendijk, J, Janssen, T.M.V. and Stokhof, M. (eds.) Formal Methods in the Study of Language, Volume 136, pp. 277-322. 1980.

Ewan Klein. VP Ellipsis in DR Theory. In J. Groenendijk, D. de Jongh and M. Stokhof, eds. Studies in Discourse Representation Theory and the Theory of Generalized Quantifiers, Foris Publications. Dordrecht, The Netherlands. 1987.

Fernando Pereira and Martha Pollack. Incremental Interpretation. Artificial Intelligence. Vol. 50. no. 1, pp. 37-82. June 1991.

Ivan A. Sag. Deletion and Logical Form. Ph.D. thesis, MIT. 1976.

Bonnie Lynn Webber. A Formal Approach to Discourse Anaphora. Ph.D. thesis, Harvard University. 1978.

Edwin Williams. Discourse and Logical Form. Linguistic Inquiry, 8(1):101-139. 1977. 